\title{
ARTÍCULOS
}

\section{Diversidad vegetal en las cuencas de los ríos Baker y Pascua (Aisén, Patagonia Chilena)}

\author{
Plant diversity in the basins of the rivers Baker and Pascua (Aisen, Chilean Patagonia)
}

\author{
Víctor Sandoval a , Carlos Ramírez ${ }^{\text {b* }}$, Cristina San Martín ${ }^{\mathrm{c}}$, Osvaldo Vidal ${ }^{\mathrm{d}}$, \\ Miguel Álvarez ${ }^{\mathrm{e}}$, Alicia Marticorena ${ }^{\mathrm{f}}$, Yéssica Pérez ${ }^{\mathrm{c}}$ \\ ${ }^{a}$ Universidad Austral de Chile, Facultad de Ciencias Forestales y Recursos Naturales, Valdivia, Chile. \\ *Autor de correspondencia: ${ }^{b}$ Pontificia Universidad Católica de Chile, Facultad de Ciencias Biológicas, \\ Departamento de Ecología, Santiago, Chile, teléfono 56-63-2206227, cramirez@uach.cl \\ ' Universidad Austral de Chile, Instituto de Ciencias de la Tierra, Valdivia, Chile. \\ ${ }^{\mathrm{d}}$ Universidad de Magallanes, Instituto de la Patagonia, Punta Arenas, Chile. \\ ${ }^{e}$ Rheinische-Friedrich Wilhelms-Universität, INRES-Vegetationsökologie, Bonn, Deutschland. \\ ${ }^{\mathrm{f}}$ Universidad de Concepción, Departamento de Botánica, Concepción, Chile.
}

\begin{abstract}
SUMMARY
Structural attributes at different levels of biological organization (species, communities, landscape) and plant biodiversity are described and compared between the basins of Baker and Pascua Rivers in Aisen Region. Four hundred and ninety four vegetation samples were taken in forest ecosystems, bush, pasture, and peat to build tables of plant communities that differentiate and characterize biodiversity. The Shannon index, frequency and phytogeographical origin of species were used. Two hundred and ninety six species distributed in 30 communities, with high diversity of plant landscapes were found. Two hundred and forty three (82\%) of these species are native, and 53 (18\%), exotic, showing high human impact. Bushes are diverse formations, with 12 communities in the Baker basin. Anthropogenic grasslands exist only in the Baker basin. The community with the highest level of species richness was the thicket of Nothofagus antarctica with 109 species, whereas the krummholz of Nothofagus pumilio presented only seven species in the Baker basin. The Shannon index shows that the Magellanic forest (Pascua basin) is the most diverse community $\left(\mathrm{H}^{\prime}=2.923\right)$, whereas the Nothofagus pumilio krummholz is the least diverse one $\left(\mathrm{H}^{\prime}=0.615\right)$. The Baker basin has a higher wealth of native and exotic species and a higher variety of plant, than hose found at the Pascua basin probably due to the intense human intervention in the former.
\end{abstract}

Key words: biodiversity index, plant communities, degradation, Aisen, Chilean Patagonia.

\section{RESUMEN}

Se describen y comparan atributos estructurales en distintos niveles de organización biológica (especies, comunidades, paisaje), de la biodiversidad vegetal en las cuencas de los ríos Baker y Pascua en la región de Aisén. Se levantaron 494 censos de vegetación en ecosistemas boscosos, arbustivos, pratenses, turbosos y esteparios para construir tablas que diferenciaran comunidades vegetales y sirvieran para caracterizar la biodiversidad. Se utilizaron la riqueza específica, índice de Shannon, frecuencia y origen fitogeográfico de las especies. Se encontraron 296 especies distribuidas en 30 comunidades, con gran diversidad del paisaje vegetal. 243 (82\%) de estas especies son nativas, mientras que 53 (18\%), exóticas, evidenciando alta antropización. Los matorrales son formaciones variadas, con 12 comunidades de la cuenca del Baker. Praderas de origen antrópico solo existen en la cuenca del Baker. La mayor riqueza específica fue la del matorral de Nothofagus antarctica con 109 especies, en tanto que el Krummholz de Nothofagus pumilio, solo presentó siete especies en ambas cuencas. El índice de Shannon muestra que la comunidad más diversa es el bosque magallánico perennifolio (cuenca Pascua) $\left(\mathrm{H}^{\prime}=2,923\right)$, mientras que el Krummholz de $N$. pumilio es la comunidad de menor diversidad $\left(\mathrm{H}^{\prime}=\right.$ 0,615). La cuenca del Baker presenta mayor riqueza de especies nativas y exóticas y mayor variedad de comunidades vegetales que la cuenca del Pascua, seguramente por intensa intervención antrópica en la primera.

Palabras clave: índices de biodiversidad, comunidades vegetales, degradación, Aisén, Patagonia chilena.

\section{INTRODUCCIÓN}

El conocimiento de la biodiversidad existente en un área determinada, es un elemento crucial cuando se precisa tomar decisiones de manejo ambiental. La biodiversidad es importante en ecosistemas naturales y seminaturales debido a que genera procesos ecológicos que, en último término, mantienen el equilibrio y la estabilidad tanto de 
los ecosistemas como de la atmósfera terrestre (Franklin 1993). Aunque existe consenso en relación a la importancia de la biodiversidad, no existe un atributo único que pueda caracterizarla globalmente (Purvis y Hector 2000). Desde el nivel genético hasta el paisajístico, existen numerosos métodos, técnicas e índices que permiten apreciar la biodiversidad; el aporte de los taxónomos y ecólogos ha sido el más significativo y ampliamente probado. Así, a partir de la información generada, las decisiones de manejo se verán fuertemente influenciadas por el nivel de conocimiento de los taxa que se evalúen, por la escala utilizada y por los atributos que puedan verse perturbados (Gaines et al. 1999).

El conocimiento de la biodiversidad es de gran importancia en territorios poco poblados y alejados de los grandes centros urbanos que necesitan desarrollarse, pero al mismo tiempo proteger su ambiente, especialmente cuando poseen recursos naturales importantes como sucede en la región de Aisén, Chile. Este estudio tiene como objetivo analizar los atributos de la biodiversidad vegetal a niveles específicos, comunitarios y paisajísticos en las cuencas de los ríos Baker y Pascua. Cuencas con marcadas diferencias de ubicación, relieve, accesibilidad, condiciones de hábitat e intervención antrópica en la provincia de Capitán Prat de la región de Aisén (Chile). Se pone énfasis en el nivel comunitario debido a que la información base proviene de tablas fitosociológicas, destinadas a estudiar y diferenciar las comunidades vegetales presentes en ambas cuencas (Dengler et al. 2008).
La hipótesis supone que, existiendo diferencias físicas, biológicas y de intervención humana muy marcadas en dichas cuencas, los atributos de biodiversidad vegetal de ambas deberían presentar grandes diferencias a todo nivel. Adicionalmente, se piensa que el elemento alóctono, especialmente la flora invasora, es el que marca la diferencia.

\section{MÉTODOS}

Lugar de trabajo. El área de estudio comprende dos importantes cuencas hidrográficas ubicadas en la región de Aisén, que tributan a los cauces de los ríos Baker y Pascua, ocupando una superficie aproximada de 150.000 ha (figura 1). La primera drena, entre otros, los grandes lagos General Carrera, Bertrand y Cochrane; mientras que la segunda, drena lagos de menor tamaño, como Berges, Gabriel Quiróz, Chico y O'Higgins. Aunque no existen estaciones climáticas en el área de estudio, es posible observar un marcado gradiente ecoclimático de dirección NE-SO. Las temperaturas medias anuales correspondientes a las estaciones más próximas fluctúan entre $10^{\circ} \mathrm{C}$ (Chile Chico, $46^{\circ} 36^{\prime}-71^{\circ} 43^{\prime}$ ) y $7,1^{\circ} \mathrm{C}$ (Puerto Edén $49^{\circ} 08^{\prime}-74^{\circ} 25^{\prime}$ ), en tanto que el rango de precipitaciones oscila entre $355 \mathrm{~mm}$ (Chile Chico) y $3.033 \mathrm{~mm}$ (Puerto Edén). Estas localidades han sido clasificadas como climas seco y ultraperhúmedo del cinturón bioclimático supratemperado, respectivamente. Este marcado gradiente determina la ocurrencia de numerosas formaciones vegetales, desde estepas hasta bosques. En el mismo sentido también es posible observar un patente gradiente

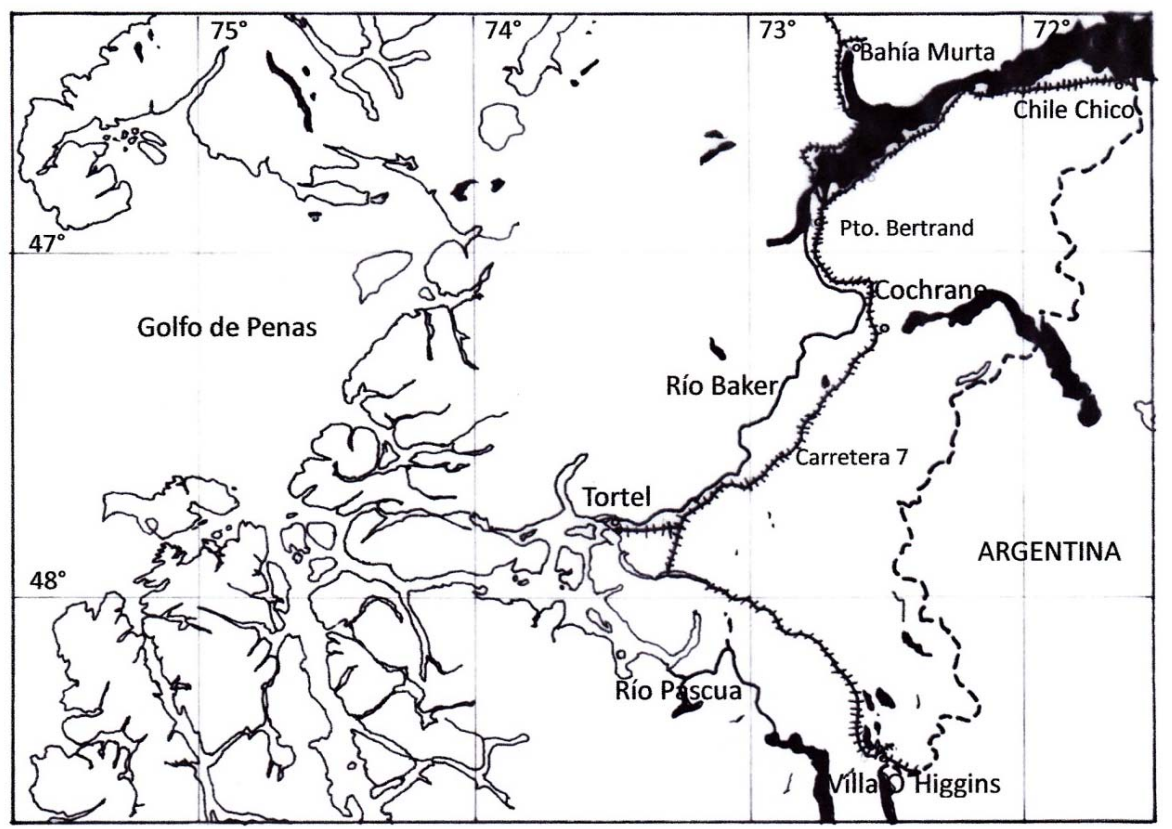

Figura 1. Ubicación de las cuencas trabajadas en la región de Aisén, Chile. Lagos y lagunas están teñidos de negro. Sólo se colocaron los ríos Baker y Pascua señalados con línea gruesa continua.

Location of the basins worked in Aisen Region, Chile. Lakes and ponds are dyed black. Only Baker and Pascua Rivers marked with thick solid line were placed. 
de perturbación antrópica, determinado aparentemente por el tamaño de los asentamientos humanos, la influencia de la actividad ganadera y la accesibilidad al área mediante rutas vehiculares. Extensas áreas boscosas han sido despejadas mediante roce por fuego para uso ganadero, siendo transformadas en praderas antropizadas. Los predios particulares no se encuentran apropiadamente delimitados, de manera que la práctica ganadera es extensiva y el ganado pasta y se mantiene en praderas, matorrales y bosques sin limitaciones, provocando grandes alteraciones en la estructura de estas formaciones vegetales (Ramírez et al. 2012).

Procedimiento de terreno. Entre los meses de abril del 2006 y enero del 2007, se organizaron cuatro campañas durante las cuales un equipo conformado por ingenieros forestales y biólogos recorrió intensamente el área de estudio. Dichas campañas fueron planificadas en un intento por inventariar con el mayor detalle la riqueza florística y vegetal del área de estudio. El difícil acceso al área de estudio obligó a los equipos a incursionarla utilizando caballos, lanchas, vehículos todo terreno y helicópteros.

Para evaluar la biodiversidad vegetal, se seleccionaron formaciones vegetales terrestres con fisonomía uniforme y en sectores con vegetación homogénea dentro de cada formación se inventarió la flora a través de parcelas de vegetación o relevès (cuadro 1) y con el análisis de la flora y su agrupamiento se define cada comunidad tomando el estatus de asociación (Dengler et al. 2008). La nomencla-

Cuadro 1. Clasificación en formaciones vegetales, nomenclatura común y científica preliminar y número de censos levantados para este trabajo de las comunidades vegetales de Aisén.

Classification in plant formations, common and scientific preliminary names and numbers of vegetation samples for this job of Aisen plant communities.

\begin{tabular}{|c|c|c|c|}
\hline Formación & Nombre común & Nombre científico & Censos \\
\hline \multirow[t]{7}{*}{ Bosques } & De coihue & Chusqueo-Nothofagetum dombeyii & 36 \\
\hline & De coihue de Chiloé & Luzuriago-Nothofagetum nitidae & 40 \\
\hline & Magallánico perennifolio & Mayteno-Nothofagetum betuloidis & 38 \\
\hline & Magallánico caducifolio & Adenocaulo-Nothofagetum pumiliae & 37 \\
\hline & Ñire en ñadi & Chusqueo-Nothofagetum antarcticae & 10 \\
\hline & De ciprés & Empetro-Pilgerodendronetum uviferae & 7 \\
\hline & Plantación & Cultivos artificiales & 7 \\
\hline \multirow[t]{12}{*}{ Matorrales } & De notro & Embothrio-Gaultherieum mucronatae & 24 \\
\hline & De yaqui & Colletietum hystrix & 14 \\
\hline & De ñire & Anemono-Nothofagetum antarcticae & 48 \\
\hline & Krummholz de lenga & Empetro-Nothofagetum pumiliae & 6 \\
\hline & Krummholz de ñire & Senecio-Nothofagetum antarcticae & 5 \\
\hline & De chapel & Escallonietum virgatae & 17 \\
\hline & De calafate & Berberidietum microphyllae & 9 \\
\hline & De chaura grande & Gaultherietum phillyreifoliae & 5 \\
\hline & De chacai & Fragario-Discarietum chacaye & 8 \\
\hline & De chilco & Berberio-Fuchsietum magallanicae & 5 \\
\hline & De rosa mosqueta & Rosetum rubiginosae & 11 \\
\hline & De pangue & No nominado aún & 7 \\
\hline \multirow[t]{5}{*}{ Praderas } & De chépica & Acaeno-Agrostietum capillariae & 8 \\
\hline & De trébol-pasto lanco & Bromo-Trifolietum repentis & 19 \\
\hline & De rime & Eleocharietum pachycarpae & 12 \\
\hline & De aira-romacilla & Airo-Rumicetum acetosellae & 11 \\
\hline & De aira-cadillo & Airo-Acaenetum integerrimae & 3 \\
\hline \multirow[t]{3}{*}{ Turberas } & Graminosa & Cortaderio-Schoenetum andinae & 30 \\
\hline & Esfagnosa & Sphagnetum magellanicii & 24 \\
\hline & Pulvinada & Drosero-Donatietum fascularis & 11 \\
\hline \multirow[t]{3}{*}{ Estepa } & De murtilla & Empetro-Gaultherietum mucronatae & 5 \\
\hline & De neneo & Mulinetum spinosae & 23 \\
\hline & De coirón & Festucetum gracillimae & 14 \\
\hline
\end{tabular}


tura de las comunidades vegetales se basa en la literatura nacional y extranjera reunida por los autores (Vidal et al. 2011). Algunos nombres se encuentran en informes del Proyecto CA 012.05 UACh-HYDROAISEN (Sandoval et al. 2007). Las parcelas consistieron en cuadrantes de $25 \mathrm{~m}^{2}$ para las praderas, estepas, turberas y mallines, 100 $\mathrm{m}^{2}$ para matorrales y $200 \mathrm{~m}^{2}$ para bosques. Estos tamaños fueron seleccionados de acuerdo a la literatura y al área mínima (Ramírez et al. 1997). Para cada parcela se determinaron en terreno las especies vegetales presentes y estimó visualmente la cobertura relativa de los individuos en conjunto de cada una de ellas, expresada en porcentaje. Cuando las especies representaban coberturas iguales o menores al $1 \%$, fueron registradas en la planilla de terreno con los signos "+", y cuando existían individuos solitarios se registraron bajo el signo " $r$ ". Para cálculos matemáticos posteriores estos signos se subieron a la unidad. El cálculo in situ de las coberturas requirió al menos la experticia de dos observadores simultáneos. En los casos en que no fue posible determinar a campo la identidad de uno o varios ejemplares, se tomaron y etiquetaron muestras de estos indicando el censo correspondiente, para ser luego herborizados, determinados en laboratorio y adicionados los nombres a posteriori al registro de los formularios de terreno.

Determinación de muestras. Las colectas herborizadas fueron determinadas y clasificadas siguiendo métodos taxonómicos tradicionales, usando claves de determinación para luego contrastarlas con ejemplares de los herbarios CONC y VALD y en la flora de Rodríguez et al. (2008). Dichos ejemplares figuran como registros en las colecciones mencionadas. La nomenclatura sigue a Zuloaga et al. (2008), actualizada periódicamente en el sitio The plant list del Jardín Botánico de Missouri (2015), USA. El origen geográfico de las especies se basa en revisión de la literatura y la proporción de especies nativas respecto a alóctonas se utilizó como indicador de pristinidad o degradación de origen antrópico. Para definir especies exóticas se consideró el criterio de Richardson et al. (2000). La presencia y abundancia de las especies vegetales en las cuencas se pueden encontrar en la publicación de Rodríguez et al. (2008).

Análisis de datos. El análisis de la diversidad se realizó de acuerdo a la escala de organización, desde el nivel comunitario con determinación de la riqueza florística, diversidad de especies según complejidad de organización vertical de las comunidades y luego entre las cuencas de los ríos Baker y Pascua.

En total se levantaron 494 censos de vegetación en formaciones vegetales terrestres correspondientes a bosques $(n=175)$, matorrales $(n=159)$, praderas $(n=53)$, turberas $(n=65)$ y estepas $(n=42)$. Los censos efectuados en mallines $(\mathrm{n}=11)$, se excluyeron del análisis, por tratarse de comunidades dulceacuícolas "azonales", es decir, no dependen del macroclima sino que de condiciones del suelo. Los censos fueron clasificados en comunidades vegetales en función de su fisonomía, de la composición florística y de especies diferenciales de acuerdo a métodos tradicionales y estadísticos modernos (Mc Cune y Grace 2002). El conjunto de censos así reunidos, se tabularon en tablas fitosociológicas separadas que fueron usadas como base de datos para analizar atributos estructurales y de composición a diferentes niveles de organización biológica (especies, comunidades, paisajístico) (Noss 1990).

Se evaluaron riqueza florística (número de especies), frecuencia, valores de importancia y origen fitogeográfico de las especies. Además, se determinó la diversidad de las comunidades expresada mediante el índice de Shannon:

$$
\mathrm{H}=-\sum_{\mathrm{i}=1}^{\mathrm{s}} \mathrm{p}(\mathrm{i}) \ln \mathrm{p}(\mathrm{i})
$$

Donde, $\mathrm{p}_{\mathrm{i}}=$ proporción de la $i$-ésima especie en una comunidad de $S$ especies.

Este índice, derivado de la teoría de la información, resume la incertidumbre o el grado de entropía que contiene un conjunto de elementos. Fue utilizado por primera vez para cuantificar la información generada por una persona que escribía sobre un teclado y actualmente se ha convertido en uno de los descriptores preferidos de biodiversidad, puesto que combina el número de especies y la abundancia relativa de ellas en una sola función matemática (Pla 2006, Badii et al. 2007, Möseler et al. 2009). Básicamente, este índice señala el grado de heterogeneidad $u$ homogeneidad de una comunidad, y advierte sobre la incertidumbre de encontrar una especie cualquiera dentro del conjunto de la comunidad. En comunidades de fisonomía homogénea, por ejemplo, donde una especie domina ampliamente y las restantes se encuentran en pequeñas proporciones, el grado de incertidumbre de encontrar alguna especie será menor (el valor del índice más bajo), que en la misma comunidad, si esta tuviese todas sus especies igualmente abundantes (en este último caso el índice presenta su máximo posible). De esta manera el indicador es muy útil para establecer comparaciones entre comunidades (Gaines et al. 1999). Para realizar estas últimas, se utilizó la columna de abundancia absoluta de las especies de cada tabla y sobre esta columna se efectuó un re-muestreo del tipo bootstrap en cada comunidad (1.000 iteraciones cada una), con la finalidad de obtener intervalos de confianza para el parámetro. Los valores así obtenidos fueron contrastados entre comunidades para obtener comparaciones estadísticamente significativas (Lanyon 1987).

Además, la riqueza florística de las formaciones vegetales fue comparada entre cuencas mediante el índice de Jaccard:

$$
I_{j}=\frac{a+b+c}{c}
$$

Donde, $\mathrm{a}=$ número de especies presentes en el sitio $\mathrm{A}, \mathrm{b}=$ número de especies presentes en el sitio $\mathrm{B}, \mathrm{y} \mathrm{c}=$ número de especies presentes en ambos sitios A y B. 
Este índice es uno de los más populares cuando se intenta comparar la riqueza específica presente entre diferentes comunidades o localidades (Badii et al. 2007).

Finalmente, se estableció una jerarquía de las comunidades basada en un dendrograma que agrupa a las comunidades de acuerdo a su similitud florística. Para ello, se elaboró una tabla de presencia-ausencia de especies que reunió a todas las comunidades y a las especies presentes en ellas (Gaines et al. 1999).

\section{RESULTADOS}

Los resultados son descritos y comparados entre cuencas hidrográficas de acuerdo a los diferentes niveles de organización biológica estudiados. Primero se describe el paisaje, luego las comunidades vegetales y finalmente los atributos referidos a especies de plantas vasculares.

Distribución de las comunidades vegetales entre cuencas. El paisaje vegetal del área de estudio es muy diverso. Las tablas fitosociológicas muestran la presencia de 30 comunidades vegetales terrestres distribuidas entre ambas cuencas, aunque existen diferencias notables en la manera como estas comunidades se distribuyen. Así por ejemplo, es posible hallar mayor diversidad comunitaria en la cuenca del Baker, mientras que en la cuenca del Pascua el paisaje vegetal es más uniforme, con menor número de comunidades. De acuerdo a esta distribución del paisaje, seis comunidades pueden considerarse comunes a ambas cuencas, mientras que 20 son exclusivas de la cuenca del río Baker y solo cuatro pueden considerarse exclusivas de la cuenca del río Pascua (cuadro 2).

Superficies de las comunidades. Los bosques representan una vasta superficie del área estudiada, más extendidos en la cuenca del Baker que en la cuenca del Pascua. Estas comunidades, se encuentran ampliamente distribuidas

Cuadro 2. Diversidad de formaciones vegetales terrestres distribuida según cuencas hidrográficas.

according to basins.

Diversity of terrestrial plant formations distributed

\begin{tabular}{lccc}
\hline \multirow{3}{*}{ Formaciones } & \multicolumn{3}{c}{ Especies } \\
\cline { 2 - 4 } & $\begin{array}{c}\text { Exclusivas } \\
\text { Baker }\end{array}$ & Compartidas & $\begin{array}{c}\text { Exclusivas } \\
\text { Pascua }\end{array}$ \\
\hline Bosques & $4(57 \%)$ & $2(29 \%)$ & $1(14 \%)$ \\
Matorrales & $9(75 \%)$ & $2(17 \%)$ & $1(8 \%)$ \\
Praderas & $5(100 \%)$ & 0 & 0 \\
Turberas & 0 & $1(33 \%)$ & $2(67 \%)$ \\
Estepas & $2(67 \%)$ & $1(33 \%)$ & 0 \\
\hline Total & $20(67 \%)$ & $6(20 \%)$ & $4(13 \%)$ \\
\hline
\end{tabular}

y representan diversos ecosistemas de composición muy diferente entre las cuencas. El bosque magallánico perennifolio, el bosque chilote y los cipresales, más higrófilos y presentes preferentemente en la cuenca del Pascua, reúnen el 10,1\% de la superficie estudiada, mientras que bosques de condiciones más secas como el bosque de coihue (Nothofagus dombeyi (Mirb.) Oerst.), el bosque magallánico caducifolio, el bosque de ñire (Nothofagus antarctica (G. Forst.) Oerst.) en suelos de ñadi (Aquands) y las plantaciones forestales, reúnen el 17,3\% de la superficie del área de estudio. Llama la atención la escasa representatividad del bosque de coihue de Chiloé (Nothofagus nitida (Phil.) Krasser), que solo ocupa una superficie de 1.321 ha (0,9\% de la superficie total).

Los matorrales son comunidades muy heterogéneas que representan el $40 \%$ de la diversidad de vegetación descrita (12 en total). Sin embargo, casi toda esta diversidad se encuentra restringida a la cuenca del río Baker. Los matorrales del Baker reúnen el 32,5 \% y los del Pascua el $0,8 \%$ de la superficie estudiada.

Las praderas, formaciones distribuidas principalmente en la cuenca del Baker, constituyen el 9,1\% de la superficie de muestreo, en tanto que turberas y estepas ocupan en conjunto el $15,4 \%$ de la superficie muestreada.

Similitud florística y comunitaria entre cuencas hidrográficas. El dendrograma basado en similitud florística indica dos grandes conglomerados, con tendencias claras a agrupar comunidades características del Baker en uno, y comunidades de la cuenca Pascua en el otro (figura 2).

El conglomerado de la cuenca Baker puede a su vez subdividirse en dos subgrupos. Uno de ellos incluye comunidades más xerofíticas como la estepa de coirón, el matorral de rosa mosqueta o la estepa de neneo (A1). Este grupo puede considerarse como representativo de la cuenca Baker Norte. El otro nodo, en tanto, agrupa comunidades un poco más higrófilas como el matorral de notro, el bosque de coihue o el bosque de ñire en ñadis, comunidades que pueden considerarse características de la cuenca Baker Sur (A2).

De la misma manera, la cuenca del Pascua, puede subdividirse en dos subconglomerados, uno agrupando a las turberas y al bosque de ciprés de las Guaitecas (B1), comunidades altamente dependientes de acumulación de agua. Las turberas de Donatia fascicularis J.R. Forst. et G. Forster y de Rime por precipitación (ombrogénicas) o acumulación de agua edáfica (topogénicas), en el caso del bosque de ciprés y de las turberas esfagnosas (B2). El otro subgrupo reúne a los bosques de condiciones higrófilas y frías, como el bosque chilote y el bosque magallánico perennifolio (B1). Este grupo incluye también posibles estados sucesionales primarios de los bosques, como los pangales y el matorral de chilco.

La comparación de la flora entre ambas cuencas, basada en el índice Jaccard, revela que estas se asemejan en solo un $61 \%$. Del total de especies registradas en las tablas 


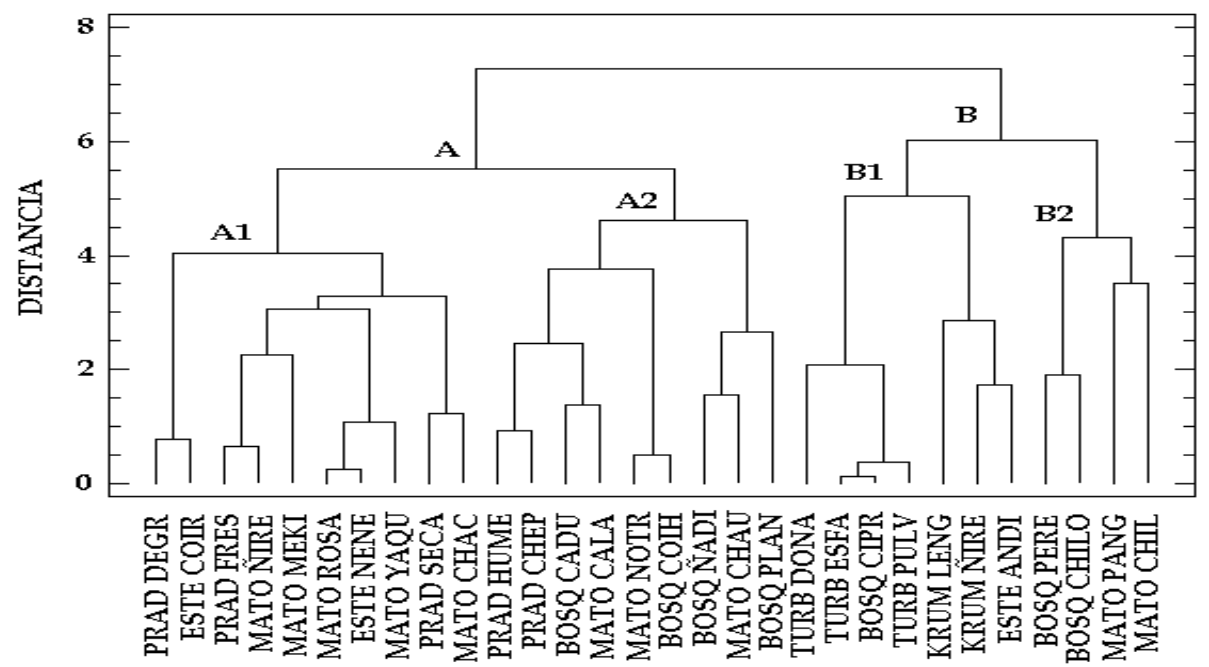

Figura 2. Dendrograma para las comunidades vegetales del área de estudio. Nótese la separación de dos conglomerados, que representan a las comunidades distribuidas principalmente en la cuenca del río Baker (A) y del río Pascua (B). Acrónimos de las comunidades: PRAD DEGR = pradera degradada, ESTE COIR = estepa de coirón, PRAD FRES = pradera fresca, MATO N NRE = matorral de ñire, MATO MEKI = matorral de meki (Chapel), MATO ROSA = matorral de rosa mosqueta, ESTE NENE = estepa de neneo, MATO YAQU = matorral de yaqui, PRAD SECA = pradera seca, MATO CHACA = matorral de chacai, PRAD HUME = pradera húmeda, PRAD CHEP = pradera de chépica, BOSQ CADU = bosque magallánico caducifolio, MATO CALA = matorral de calafate, MATO NOTR = matorral de notro, BOSQ COIH = bosque de coihue, BOSQ ÑAD = matorral de ñire en ñadi, MATO CHAU = matorral de chaura grande, BOSQ PLAN = plantación forestal, TURB DONA = turbera de Donatia, TURB ESFA = turbera esfagnosa, BOSQ CIPR $=$ bosque de ciprés, TURB PULV $=$ turbera pulvinada, KRUM LENG $=$ Krummholz lenga, KRUM ÑIRE $=$ Krummhoz ñire, ESTE ANDI = estepa andina, BOSQ PERE = bosque magallánico perennifolio, BOSQ CHILO = bosque chilote, MATO PANG = matorral pangal, MATO CHIL = matorral de chilco.

Dendrogram of plant communities in the study area. The separation of two conglomerates, representing mainly communities found in the watershed of Baker River (A) and in that of Pascua River (B), is noteworthy. Acronyms of communities: PRAD DEGR = degraded prairie, THIS COIR $=$ coirón steppe, PRAD FRES $=$ fresh meadow, MATO ÑIRE $=$ ñire scrub, MATO MEKI = meki (chapel) scrub, MATO ROSA = wild rose scrub, ESTE $\mathrm{NENE}=$ neneo steppe, MATO YAQU $=$ yaqui scrub, PRAD DRY = dry meadow, MATO CHACA = chacai scrub, PRAD HUME = wet meadow, PRAD $\mathrm{CHEP}=$ chépica prairie, $\mathrm{BOSQ} \mathrm{CADU}=$ Magellanic deciduous forest, $\mathrm{MATO}$ CALA $=$ calafate scrub, $\mathrm{MATO}$ NOTR $=$ notro $\mathrm{scrub}, \mathrm{BOSQ} \mathrm{COIH}=$ coihue forest, BOSQ ÑADI = ñire scrub in Nadi, MATO CHAU = big chaura scrub, BOSQ PLAN = forest plantation, DONA TURB = Donatia bog, TURB ESFA = Sphagnum bog, BOSQ CIPR = cypress forest, TURB PULV = pulvinate bog, KRUM LENG = lenga krummholz, KRUM ÑIRE $=$ ñire krummhoz, ESTE ANDI = Andean steppe, BOSQ PERE = evergreen Magellanic forest, BOSQ CHILO = Chilote forest, MATO PANG = pangal scrub, MATO CHIL $=$ chilco scrub.

fitosociológicas, 12 se encontraron exclusivamente en la cuenca del Pascua, 102 especies solo se encontraron en la cuenca Baker y 182 especies fueron compartidas por ambas cuencas.

Biodiversidad a nivel comunitario. Se detectó la presencia de 30 comunidades vegetales distribuidas a través de las cuencas. De las 296 especies presentes en las tablas fitosociológicas, $243(82 \%)$ son nativas y 53 (18\%), exóticas.

Riqueza florística en las comunidades. Se encontraron 172 especies en las formaciones de bosque, de las cuales 24(14\%) son exóticas (cuadro 3).

El bosque de coihue (cuenca Baker) es el que posee mayor riqueza específica, con 83 especies, le sigue el bosque magallánico caducifolio, con 79 especies. Los bosques del Pascua, en tanto, poseen riquezas levemente menores, pero casi carecen de especies exóticas. El bosque chilote
Cuadro 3. Riqueza de especies para las formaciones vegetales terrestres presentes en el área de estudio.

Species richness for terrestrial plant communities in the study area.

\begin{tabular}{lcccc}
\hline & & \multicolumn{3}{c}{ Especies } \\
\cline { 3 - 5 } Formación & Censos & Nativas & Exóticas & Total \\
\hline Bosques & 175 & $148(86 \%)$ & $24(14 \%)$ & $172(100 \%)$ \\
Matorrales & 159 & $148(76 \%)$ & $48(24 \%)$ & $196(100 \%)$ \\
Praderas & 53 & $63(60 \%)$ & $42(40 \%)$ & $105(100 \%)$ \\
Turberas & 65 & $92(99 \%)$ & $1(1 \%)$ & $93(100 \%)$ \\
Estepas & 42 & $87(74 \%)$ & $30(26 \%)$ & $117(100 \%)$ \\
\hline Total & 495 & $237(82 \%)$ & $53(18 \%)$ & $290(100 \%)$ \\
\hline
\end{tabular}


con 74 especies solo posee dos especies exóticas, mientras que el bosque magallánico perennifolio, con 73 especies, no presenta especies exóticas.

Los matorrales representan las formaciones con mayor número de especies (cuadro 4). Aunque el número total de especies es alto, existen diferencias notables en la riqueza florística de las comunidades arbustivas.

El matorral de ñire es el que presenta la mayor riqueza específica (109 especies), tanto de nativas como de exóticas. Otras comunidades con alta riqueza de especies distri-

Cuadro 4. Riqueza total de especies $(\mathrm{R})$ e índice de Shannon $(\mathrm{H})$ calculado por re-muestreo, para las comunidades terrestres evaluadas en el presente estudio. $\mathrm{IC}=$ Intervalo de confianza.

Total species richness $(\mathrm{R})$ and Shannon index $(\mathrm{H})$ calculated by re-sampling, for terrestrial communities evaluated in the present study. $\mathrm{IC}=$ confidence interval

\begin{tabular}{|c|c|c|c|c|c|c|c|}
\hline \multirow[b]{2}{*}{$\mathrm{N}^{\circ}$} & \multirow{2}{*}{$\begin{array}{l}\text { Formación } \\
\text { Comunidad }\end{array}$} & \multirow[b]{2}{*}{ Cuenca } & \multirow[b]{2}{*}{ Censos } & \multirow[b]{2}{*}{$\mathrm{R}$} & \multirow[b]{2}{*}{$\mathrm{H}$} & \multicolumn{2}{|c|}{ Límites del IC } \\
\hline & & & & & & Inferior & Superior \\
\hline \multicolumn{8}{|c|}{ Bosques } \\
\hline 1 & De coihue & Baker & 36 & 83 & 1,986 & 1,922 & 2,032 \\
\hline 2 & De coihue de Chiloé & Baker/Pascua & 40 & 74 & 2,805 & 2,769 & 2,832 \\
\hline 3 & Magallánico perennifolio & Pascua & 38 & 73 & 2,923 & 2,888 & 2,947 \\
\hline 4 & Magallánico caducifolio & Baker & 37 & 79 & 2,426 & 2,367 & 2,463 \\
\hline 5 & De ñire en Ñadi & Baker & 10 & 33 & 2,169 & 2,091 & 2,223 \\
\hline 6 & De ciprés & Baker/Pascua & 7 & 42 & 2,463 & 2,370 & 2,513 \\
\hline 7 & Plantación & Baker & 7 & 14 & 1,240 & 1,169 & 1,292 \\
\hline \multicolumn{8}{|c|}{ Matorrales } \\
\hline 8 & De notro & Baker & 24 & 61 & 2,188 & 2,126 & 2,227 \\
\hline 9 & De yaqui & Baker & 14 & 39 & 1,988 & 1,903 & 2,042 \\
\hline 10 & De ñire & Baker & 48 & 10 & 2,750 & 2,691 & 2,785 \\
\hline 11 & Krummholz de lenga & Baker & 6 & 7 & 0,615 & 0,525 & 0,689 \\
\hline 12 & Krummholz de ñire & Pascua & 5 & 12 & 1,328 & 1,259 & 1,386 \\
\hline 13 & De chapel & Baker & 17 & 74 & 2,211 & 2,115 & 2,268 \\
\hline 14 & De calafate & Baker & 9 & 49 & 2,382 & 2,285 & 2,436 \\
\hline 15 & De chaura grande & Baker & 5 & 43 & 2,523 & 2,397 & 2,580 \\
\hline 16 & De chacai & Baker & 8 & 40 & 2,145 & 2,054 & 2,203 \\
\hline 17 & De chilco & Baker/Pascua & 5 & 35 & 2,214 & 2,089 & 2,287 \\
\hline 18 & De rosa Mosqueta & Baker & 11 & 60 & 2,423 & 2,311 & 2,483 \\
\hline 19 & De pangue & Baker/Pascua & 7 & 26 & 1,311 & 1,143 & 1,427 \\
\hline \multicolumn{8}{|c|}{ Praderas } \\
\hline 20 & De chépica & Baker & 8 & 25 & 1,756 & 1,646 & 1,843 \\
\hline 21 & De trébol-pasto lanco & Baker & 19 & 65 & 2,769 & 2,697 & 2,810 \\
\hline 22 & De rime & Baker & 12 & 45 & 2,472 & 2,381 & 2,522 \\
\hline 23 & De aira-romacilla & Baker & 11 & 58 & 2,108 & 1,978 & 2,170 \\
\hline \multirow[t]{2}{*}{24} & De aira-cadillo & Baker & 3 & 20 & 1,946 & 1,772 & 2,047 \\
\hline & \multicolumn{7}{|l|}{ Turberas } \\
\hline 25 & Graminosa & Pascua & 30 & 71 & 2,322 & 2,255 & 2,369 \\
\hline 26 & Esfagnosa & Baker/Pascua & 24 & 66 & 2,038 & 1,970 & 2,091 \\
\hline \multirow[t]{2}{*}{27} & Pulvinada & Pascua & 11 & 33 & 2,224 & 2,129 & 2,288 \\
\hline & Estepas & & & & & & \\
\hline 28 & De murtilla & Baker/Pascua & 5 & 32 & 1,800 & 1,636 & 1,906 \\
\hline 29 & De neneo & Baker & 23 & 84 & 2,539 & 2,456 & 2,591 \\
\hline 30 & De coirón & Baker & 14 & 37 & 1,865 & 1,771 & 1,936 \\
\hline
\end{tabular}


buidas en la cuenca del Baker son la estepa de neneo (84 especies) y el bosque de coihue (83 especies).

Contrariamente a lo anterior, los matorrales achaparrados presentes sobre el límite altitudinal de los bosques (Krummholz), representan las comunidades más pobres en especies. Ninguna de las especies que componen estos matorrales es exótica.

Las praderas poseen valores variables para la riqueza florística, aunque siempre con una alta presencia de exóticas. Estas formaciones se encuentran restringidas de preferencia en la cuenca del Baker. Las praderas con mayor riqueza son la pradera de trébol-pasto lanco (fresca) y de aira-romacilla (seca). Las praderas degradadas (aira-cadi1lo) y de chépica, solo poseen 25 y 20 especies, respectivamente.

Las turberas llaman la atención debido al alto número de especies que poseen. Se trata de hábitat extremos en los que, sin embargo, ha sido posible encontrar una diversidad muy alta que supera la conocida para el área. La turbera pulvinada (cuenca Pascua) posee 71 especies, todas de carácter nativo, lo que revela su condición prístina. La turbera esfagnosa, presente en ambas cuencas, posee también alta riqueza, con 66 especies de las cuales solo una Festuca rubra L. es exótica, revelando igualmente un estado más bien primario. Finalmente, la turbera pulvinada de $D$. fascicularis, que se distribuye formando mosaicos junto a la turbera esfagnosa, es más pobre en especies. En esta comunidad se observaron 33 especies, todas nativas.

Las estepas poseen números variables de riqueza florística. La estepa de neneo contiene el mayor número de especies, 84 que representan uno de los valores más altos hallados en este estudio para una comunidad. La estepa de neneo (Mulinum spinosum (Cav.) Pers.), al igual que mu- chas otras comunidades de la cuenca del Baker, se encuentran altamente degradadas como lo demuestra la alta presencia de exóticas. La estepa de coirón, en tanto, presenta valores intermedios con 37 especies. Contrariamente, la estepa altoandina de Empetrum rubrum Vahl. ex Willd., distribuida sobre el límite altitudinal de los bosques, presenta valores relativamente bajos para la riqueza de especies. Se encontraron 32 especies, muchas de las cuales son exclusivas de esta comunidad. No hubo especies exóticas.

Diversidad de especies en las comunidades. Los valores obtenidos para el índice de Shannon, son presentados considerando las características fisonómicas de la vegetación referidas a estratificación vertical (comunidades monoestratificadas, biestratificadas y pluriestratificadas) y luego, según la cuenca hidrográfica en que estas comunidades se distribuyen (figura 3).

Los bosques pluriestratificados de la cuenca del Pascua son más diversos que aquellos de la cuenca del Baker, es decir, en general existen más especies y estas se encuentran también más equitativamente distribuidas en el espacio en la primera de las cuencas. Los bosques magallánico perennifolio y chilote son particularmente diversos, presentando los mayores índices con valores de 2,923 y 2,805 , respectivamente (cuadro 5). Los bosques del Baker, en tanto, presentan una menor diversidad, con valores Shannon que fluctúan entre 1,240 para las plantaciones de pino (Pinus spp.) y 2,426 para el bosque magallánico caducifolio.

Los matorrales en la cuenca Baker presentan alta diversidad, comparados con aquellos presentes en la cuenca del Pascua. El matorral de ñire es particularmente heterogéneo, con valores Shannon que casi alcanzan a los de los bosques de la cuenca del Pascua. Muy posiblemente

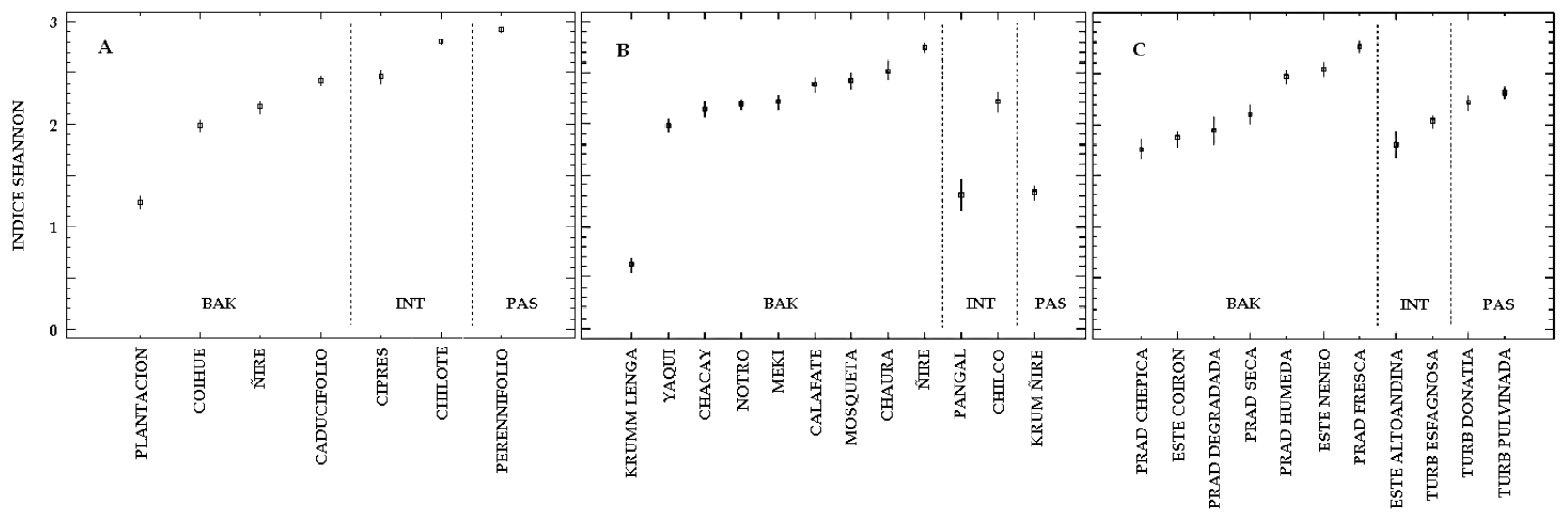

Figura 3. Índice de Shannon obtenidos por bootstrapping para las comunidades vegetales del área de estudio. A= comunidades pluriestratificadas (e.g. bosques), $\mathrm{B}=$ biestratificadas (e.g. matorrales) y $\mathrm{C}=$ monoestratificadas (e.g. estepas, praderas, turberas), de las cuencas del río Baker (BAK), comunes entre ambas cuencas (INT) y de la cuenca del Pascua (PAS). Las barras representan el intervalo de confianza de la media.

Shannon index obtained by bootstrapping for plant communities in the study area. A = pluristratified communities (e.g. forests); $\mathrm{B}=$ biestratified (e.g. bushes) and $\mathrm{C}=$ monostratified (e.g. steppes, meadows, bogs) in Baker River basins (BAK), in the two basins (INT) and in the Pascua basin (PAS). Bars represent confidence interval around the mean. 
Cuadro 5. Riqueza de especies para las formaciones vegetales según la cuenca hidrográfica estudiada.

Species richness to the plant formations according to the studied watershed.

\begin{tabular}{lcccccc}
\hline & \multicolumn{3}{c}{ Cuenca del Baker } & \multicolumn{3}{c}{ Cuenca del Pascua } \\
\cline { 2 - 6 } Formación & Nativas & Exóticas & Total & Nativas & Exóticas & Total \\
\hline Bosques & $132(85 \%)$ & $24(15 \%)$ & $156(100 \%)$ & $108(92 \%)$ & $9(8 \%)$ & $117(100 \%)$ \\
Matorrales & $143(75 \%)$ & $48(25 \%)$ & $191(100 \%)$ & $69(78 \%)$ & $19(22 \%)$ & $88(100 \%)$ \\
Praderas & $63(60 \%)$ & $42(40 \%)$ & $105(100 \%)$ & - & - & - \\
Turberas & $65(98 \%)$ & $1(2 \%)$ & $66(100 \%)$ & $92(99 \%)$ & $1(1 \%)$ & $93(100 \%)$ \\
Estepas & $87(74 \%)$ & $30(26 \%)$ & $117(100 \%)$ & $32(100 \%)$ & 0 & $32(100 \%)$ \\
\hline Total & $232(81 \%)$ & $53(19 \%)$ & $285(100 \%)$ & $173(89 \%)$ & $22(11 \%)$ & $195(100 \%)$ \\
\hline
\end{tabular}

la alta riqueza de exóticas participa en un incremento del índice. En general, las comunidades arbustivas del Baker son muy diversas, presentando altas riquezas de especies y abundancias equitativas de las mismas. Otras comunidades diversas son el matorral de chaura grande (Gaultheria phillyreifolia (Pers.) Sleumer), el matorral de rosa mosqueta (Rosa rubiginosa L.) y el matorral de calafate (Berberis microphylla $\mathrm{G}$. Forst.), con valores para el índice de Shannon de 2,523, 2,423 y 2,382, respectivamente.

Contrariamente, las comunidades arbustivas del Pascua presentan valores bajos para el índice. El matorral de chilco (Fuchsia magellanica Lam.), aunque es el más diverso dentro de la cuenca Pascua, alcanza un valor Shannon de 2,214. El Krummholz de ñire y el pangal, en tanto, son comunidades muy poco diversas.

Las comunidades monoestratificadas, como las praderas, estepas o turberas, presentan valores variables para el índice de Shannon. No parece existir relación entre la distribución de las comunidades y su diversidad. En la cuenca del Baker, la pradera fresca, la estepa de neneo y la pradera húmeda presentan los valores más altos para la métrica, con índices de 2,769, 2,539 y 2,471, respectivamente. En todos los casos se trata de hábitats de origen antrópico y altamente degradados, en donde la presencia de exóticas es notable. Las comunidades con menor diversidad son la pradera de chépica, la estepa de coirón y la estepa altoandina de Empetrum rubrum, con valores de 1,756, 1,865 y 1,800 , respectivamente. El anexo 1 muestra la significancia de todo este análisis.

Biodiversidad a nivel especifico. El cuadro 5 muestra que, en general, las comunidades de la cuenca Baker tienen mayor riqueza de especies que las comunidades de la cuenca Pascua. Sin embargo, esta diversidad muestra también alta intervención antrópica, la que se verifica con la presencia de especies introducidas. Las praderas, matorrales y estepas del Baker tienen una alta riqueza de especies exóticas. Las comunidades del Pascua, en tanto, con menor riqueza, presentan menor grado de perturbación. Sin embargo, las comunidades pioneras en derrumbes, como los pangales, presentan una alta riqueza de exóticas.

Especies más representativas y escasas. Pocas especies vasculares son frecuentes en las distintas comunidades: $B$. microphylla, $H$. lanatus, $N$. antarctica, Taraxacum officinale Weber ex F. H.Wigg. y Acaena ovalifolia Ruiz et Pav. se encontraron en 24, 21, 21, 20 y 19 de las 30 comunidades vegetales terrestres evaluadas, respectivamente.

Especies exóticas con potencial invasor. Las plantas exóticas se encuentran ampliamente distribuidas en el área de estudio, pero son particularmente frecuentes y abundantes en la cuenca del río Baker. Las especies de plantas exóticas más ampliamente distribuidas en el área de estudio son $H$. lanatus, T. officinale, Trifolium repens L., Hypochaeris radicata L.y Rumex acetosella L. apareciendo en 22, 20, 19, 17 y 17 de las 30 comunidades vegetales evaluadas.

\section{DISCUSIÓN}

Las diferencias encontradas a todo nivel en la biodiversidad de las dos cuencas estarían determinadas por gradientes eco-climáticos y de intervención humana. La cuenca del Pascua, más húmeda y fría, presenta menor diversidad de comunidades y menor grado de intervención antrópica. En tanto la cuenca del Baker, más cálida y seca, presenta mayor diversidad de comunidades y mayor perturbación antrópica (Ramírez et al. 2012, San Martín et al. 2014).

Las cuencas estudiadas poseen claras diferencias físicas y de actividad antrópica que determinan cambios apreciables en la composición florística de las comunidades. Muchas especies tienen rangos ecológicos restringidos, lo que tiene que ver con la abundancia relativa de las comunidades donde viven y que representan sus hábitats (Ramírez et al. 2015). Hay que considerar, sin embargo, el reducido tamaño de las comunidades arbustivas del Pascua, como los matorrales de chilco y los pangales, que posiblemente 
representan estadios sucesionales primarios naturales de comunidades boscosas presentes en dicha cuenca (Hildebrand-Vogel 1988). El Krummholz de ñire y el pangal, además, son comunidades poco diversas, debido a las limitantes impuestas por la altitud y a la características pioneras que poseen, respectivamente. Especies frecuentes en las comunidades son muy plásticas en sus hábitos y se encuentran ampliamente distribuidas en el área de estudio. Por el contrario, 76 especies que aparecen sólo en una comunidad vegetal dan cuenta de una restringida amplitud de nicho.

Las perturbaciones antrópicas pueden no solo incrementar la riqueza específica de las comunidades, sino también su diversidad o heterogeneidad estructural natural como lo demuestran los resultados. Esto está claramente relacionado con la historia antrópica de perturbaciones a las que los hábitats naturales han estado sometidos y a las actividades que el hombre ha practicado en estas vastas extensiones en pos de la colonización, tal como ha ocurrido en Patagonia Austral (Mermoz et al. 2005, Vidal 2012). Actividades como roce a fuego de los bosques, práctica ganadera extensiva o tala de árboles para combustible o material de construcción, han cambiado drásticamente la estructura y composición de las comunidades nativas, provocando entre otros procesos, la pérdida de especies nativas, la introducción de especies exóticas y el incremento de las invasiones biológicas. Especialmente importante resulta la intervención voluntaria destinada a "limpiar el bosque" es decir, eliminar el sotobosque para introducir ganado al bosque que ahora actúa como un "silvo-pastoreo", en el cual se forma una cubierta herbácea basada en especies pratenses introducidas de origen europeo (San Martín et al. 2014). Este fenómeno se presentó en ambas cuencas, aunque fue muy claro en los bosques de coihue y magallánico caducifolio en la del Baker, evidenciando mayor impacto antrópico.

Llama la atención la alta diversidad de paisajes arbustivos, los cual posiblemente se relacionan con diferentes grados de perturbación humana y con la agresividad de los dominantes que poseen lignotúberes de gran resistencia y larga vida. La alta presencia de especies exóticas en los matorrales los caracteriza como comunidades secundarias, resultantes de la degradación de los bosques. De lo anterior se deduce que las actividades humanas provocan tal presión sobre el paisaje vegetal, que lo diversifican estructuralmente (Roig et al. 1985, Ramírez et al. 2012).

En la región investigada es posible apreciar procesos de invasiones biológicas promovidas por especies de plantas exóticas como el caso de los matorrales de rosa mosqueta, que corresponden a comunidades terciarias altamente degradadas (Fuentes et al. 2014)), es decir, son comunidades que se forman sobre comunidades secundarias (e.g. praderas), con suelos degradados por el exceso de pastoreo (Richardson et al. 2000). Sin embargo, es difícil en este estudio adjudicar o predecir los efectos que estas invasiones biológicas están provocando en los ecosistemas, aunque algunas especies, como R. rubiginosa, están transformando severa y aceleradamente el paisaje vegetal. Esta especie muestra una extraordinaria capacidad de dispersión endozoócora, al ser comidos los frutos por caballos y no sufrir deterioro las semillas en su paso por el tracto digestivo (Ramírez et al. 2012).

Hay que destacar la condición prístina en que se encuentran los bosques chilotes y magallánicos perennifolio, careciendo de especies exóticas y de perturbaciones antrópicas mayores debido, por una parte, a la altitud en que estos hábitats se encuentran (entre 900 y 1.200 m s.n.m) y por otra, a la imposibilidad de acceso por parte del hombre y su ganado, lo cual limita el ingreso de propágulos desde áreas de infestación. Es posible, incluso, que ni siquiera estos propágalos estén presentes en estas comunidades. Sin embargo, también hay un efecto importante de los caminos, más frecuentes en la cuenca del Baker, que cruzan o bordean los rodales, transformándose en vías eficientes para la dispersión de diásporas de malezas (Vidal 2005). La escasez del bosque Chilote en la cuenca poco intervenida del Pascua, se debe seguramente a una distribución natural de esta comunidad que requiere condiciones más templadas y húmedas como son las costeras donde ella abunda. Las plantaciones jóvenes de Pinus spp. tienen alto valor de diversidad, lo que posiblemente se debe a las condiciones más luminosas del sotobosque debido a la baja cobertura arbórea, que favorecen el desarrollo de plantas heliófilas, que estarían contribuyendo a incrementar del índice.

El hecho de que las turberas hayan presentado una alta riqueza específica puede deberse a que dadas las condiciones climáticas de toda la región estudiada, existan muchas especies herbáceas nativas susceptibles de colonizar esas áreas. Sin embargo, tampoco se puede descartar un efecto de muestreo que privilegió estas formaciones debido a su especial naturaleza y ecología que permiten una fácil diferenciación de ellas en el paisaje. Se ha probado que el número de especies de una comunidad aumenta con el número de censos (Ramírez et al. 2012). Lo mismo se comprueba, pero a la inversa, con la poca riqueza específica de los "pangales" que se forman en derrumbes naturales o artificiales provocados durante la construcción de caminos, biotopos poco abundantes en la región estudiada. En el caso de la estepa de Mulinum spinosum (neneo) habría que considerar que, según Ramírez et al. (2012), ella podría tener un origen primario en la estepa oriental, pero un origen secundario en el centro de la región investigada.

\section{AGRADECIMIENTOS}

Investigación realizada en el marco del Proyecto CA 012.05 UACh-HYDROAISEN

\section{REFERENCIAS}

Amigo J, C Ramírez. 1998. A bioclimatic classification of Chile: woodlands communities in the temperate zone. Plant Eco- 
logy 136: 9-26.

Badii M, J Landeros, E Cerna. 2007. Patrones de asociación de especies y sustentabilidad. Daena: International Journal of Good Conscience 3(1): 632-660.

Dengler J, M Chytry, J Ewald. 2008. Phytosociology. p. 27672779. In Jøgensen SE, BD Fath eds. Encyclopedia of Ecology. Oxford, England. Elsevier. p. 2767-2779.

Gaines W, R Harrod, F Lehmkuhl. 1999. Monitoring biodiversity: quantification and interpretation. Portland, USA. Department of Agriculture Forest Service. 27 p.

Franklin J. 1993. Preserving biodiversity: species, ecosystems or landscapes? Ecological Applications 3: 202-205.

Fuentes N, P Sánchez, A Pauchard, J Uuurutia, L Cavieres, A Marticorena. 2014. Plantas invasoras del Centro-Sur de Chile: Una Guía de Campo. Concepción, Chile. Laboratorio de Invasiones biológicas. $275 \mathrm{p}$.

Hildebrand-Vogel R. 1988. Ersatzgesellschaften des nordwestpatagonischen Lobeerwaldes in Chile zwischen 42 und $45^{\circ} \mathrm{s}$. Br. Flora 180: 171-176.

Knapp R. 1984. Considerations on quantitative parameters and qualitative attibutes in vegetation analysis and in phytosociological relevés. In Knapp R ed. Sampling methods and taxon analysis in vegetation science. La Haya, Holanda. Junk Publishers. p. 77-119.

Lanyon S. 1987. Jackknifing and bootstrapping: important "new" statistical techniques for ornithologists. The Auk 104: 144156.

Mc Cune B, G Grace. 2002. Analysis of ecological communities. Gleneden Beach, USA. MjM Sofware Design. 304 p.

Mermoz M, T Kitzberger, T Veblen. 2005. Landscapes influences and occurrence of wild fire spread in Patagonian forests and shrublands. Ecology 86: 2705-2715.

Missouri Botanical Garden. 1915. The plant list. Consultado 24 sept. 2015. Disponible en http://www.theplantlist.org.

Möseler B, R Sievers, M Álvarez. 2009. Diversitätsanalyse unter Verwendung des Programmes DiversityCalc am Beispiel eines Wald-Transektes vom Aremberg in der Osteifel. Decheniana 162: 67-78.

Noss RF. 1990. Indicators for monitoring biodiversity: a hierarchical approach. Conservation Biology 4: 355-364.

Pla L. 2006. Biodiversidad: Inferencia basada en el índice de Shannon y la riqueza. Interciencia 31: 583-590.

Purvis A, A Hector. 2000. Getting the measure of biodiversity. Nature 405: 212-219.

Ramírez C, C San Martín, P Ojeda. 1997. Muestreo y tabulación fitosociológica aplicados al estudio de los bosques nativos.
Bosque 18(2): 19-27.

Ramírez C, V Sandoval, C San Martín, M Álvarez, Y Pérez, C Novoa. 2012. El paisaje rural antropogénico de Aisén, Chile: Estructura y dinámica de la vegetación. Gayana Botanica 69(1): 219-231.

Ramírez C, V Sandoval, JC Cisternas, H Rivera, O Vidal, C San Martín, M Alvarez, Y Pérez. 2015. Utilizando la metodología fitosociológica para diferenciar hábitats: un ejemplo con especies chilenas de Schinus (Anacardiaceae) en la Región de Aisén, Chile. Gayana Botanica 72(2): 350-366.

Richardson D, P Pysek, M Rejmánek, M Barbour, F Panetta, C West. 2000. Naturalization and invasion of alien plants: concepts and definitions. Diversity and Distributions 6: 93-107.

Rodríguez R, A Marticorena, E Teneb. 2008. Plantas vasculares de los ríos Baker y Pascua, Región de Aisén, Chile. Gayana Botanica 65(1): 39-70.

Roig F, J Anchorena, O Dollenz, A Faggi, E Méndez. 1985. Las comunidades vegetales de la transecta botánica de la patagonia austral. In Boelcke O, D Moore, F Roig eds. Transecta Botánica de la Patagonia Austral. Buenos Aires, Argentina. Instituto Salesiano de Artes Gráficas. p. 350-456.

San Martín C, V Sandoval, M Álvarez, O Vidal, Y Pérez, J Solís. 2014. Comparación de etapas de degradación vegetacional con manejo pecuario utilizando valores bioindicadores de Ellenberg en la Patagonia Chilena. Bosque 35(2): 141-154.

Sandoval V, C Ramírez, R Rodríguez. 2007. Determinación de la línea base de vegetación y flora terrestre para el Proyecto Hidroeléctrico Aysén. Valdivia, Chile. Facultad de Ciencias Forestales, Universidad Austral de Chile. 234 p.

Vidal O. 2005. Flora exótica adyacente a senderos remotos en el Parque Nacional Torres del Paine (Magallanes, Chile). Tesis Licenciatura en Ciencias Biológicas. Valdivia, Chile. Facultad de Ciencias, Universidad Austral de Chile. 124 p.

Vidal O. 2012. Anthropogenic disturbances affecting southern beech (Nothofagus pumilio) forests in Torres del Paine Biosphere Reserve, southern Chilean Patagonia. Doctoral thesis. Freiburg, Germany. Faculty of Forests and Environmental Sciences, Albert-Ludwigs University. 122 p.

Vidal O, JR Bannister, V Sandoval, Y Pérez, C Ramírez. 2011. Woodland communities in the Chilean cold-temperate zone (Baker and Pascua basins): Floristic composition and morpho-ecological transition. Gayana Botanica 68(2): 141154.

Zuloaga F, O Morrone, M Belgrano. 2008. Catálogo de las plantas vasculares del cono sur. Missouri, USA. Missouri Botanical Garden Press. 3348 p. 
